\title{
Phase transitions of a neutral polyampholyte
}

\author{
Marcia C. Barbosa*, Yan Levin \\ Instituto de Fisica, Universidade Federal do Rio Grande do Sul Caixa Postal 15051, CEP 91501-970, \\ Porto Alegre, RS, Brazil
}

Received 20 January 1996

\begin{abstract}
The behavior of a neutral polyampholyte, PA, chain with $N$ monomers is analyzed in the framework of Flory's affine network theory of rubber elasticity. The PA chain, in addition to the neutral monomers, also contains an equal number of positively and negatively charged monomers. In order to account for the electrostatic interaction, the Debye-Hückel theory augmented by the Bjerrum's ideas of dipolar formation is used. We find that at high temperatures the chain exists in a dilute collapsed state with a radius of gyration scaling as $N^{v}$ with $v=\frac{1}{3}$ (microelectrolyte). For low temperatures the PA resembles an affine network (microgel) with $v=\frac{2}{5}$. In the $\theta$ solvent, $v=\frac{3}{8}$. If the quality of the solvent is decreased, the microgel undergoes a collapse transition into a compact globular state. The high and low temperature regimes are separated by a first-order phase transition. The full phase-diagram of the PA is calculated as a function of temperature and quality of the solvent.
\end{abstract}

\section{Introduction}

The study of physical properties of neutral polymers has received a lot of attention in the last two decades. In particular, it was found that these macromolecules can exhibit a transition from an extended coil to a compact globular state, and that this transition is related to the folding transition in proteins [1].

From a theoretical point of view, this transition is well understood in terms of scaling laws that associate the coil-globular transition with a tricritical behavior similar to the one present in magnetic systems. Within this approach, conformations of the homopolymers are characterized by a critical exponent $v$, which relates the root-meansquare size of the polymer with the number of monomers, $N, R_{\mathrm{G}} \approx a N^{\nu}$, where $a$ is the average separation between the monomers. For good solvents, the repulsive excluded volume interaction between the monomers leads to $v \approx 0.588$, in three dimensions. In

\footnotetext{
${ }^{*}$ Corresponding author.
} 
a poor solvent, there is an effective attractive attraction between the monomers which leads to the collapse of the polymer and to $v=\frac{1}{3}$. A change in temperature or the addition of a specific solvent can induce a transition between these two conformations. At the transition temperature, $T_{\theta}, v=\frac{1}{2}[4,5]$.

In many situations of chemical and biological importance some of the monomers of a polymer can become ionized. The resulting Coulombic interaction between the charged monomers strongly effects the conformational properties of a polymer. In this case, the standard methods developed for the study of homopolymers become inapplicable.

There are two distinct classes of charged polymers: polyelectrolytes and polyampholytes. Polyelectrolytes are polymers made of charged monomers all of which carry a charge of the same sign. A common example of a polyelectrolyte is a DNA dissolved in water. In these molecules the strong Coulombic repulsion between the charges along the chain forces the polyion to assume an extended "rodlike" configuration [6-10] .

Polyampholytes (PA) are polymers that, besides neutral monomers, also contain a sequence of positively and negatively charged groups. Since proteins are a special class of PA, it is important to obtain a deeper insight into the physics of these complex molecules. Due to the difficulties of taking a proper account of the Coulomb interactions present in the PA, our understanding of this class of polymers is rather poor.

The first attempt at describing the coil-globule transition in a neutral PA (equal number of positive and negative monomers) was carried out by Edwards et al. [11]. They argued that the net electrostatic energy of a PA molecule is attractive and forces the molecule to be more compact. This, however, is counteracted by a reduction in entropy of the chain due to the confinement within a sphere smaller than its natural radius. This competition leads to a transition from an extended coil to a "dilute globular state". The dilute globular state, or a microelectrolyte, is characterized by a density smaller than that of the collapsed state of a chain made of monomers whose groups interact only through a short-range force.

Higgs and Joanny [12] extended the Edwards et al. theory, including repulsive forces due to the excluded volume interactions. For dilute solvents, they found that small chains, with $N<N_{1}$ exist in an extended, or a coil state depending on the quality of the solvent, while very long chains, with $N>N_{1}$, are always collapsed. In this last case, small parts of the chain, the blobs, with the number of monomers less than $N_{1}$, retains the coil configuration, while on larger scales the globule is composed of a closely packed array of blobs.

On the basis of Monte Carlo simulations, Victor and Imbert [13] questioned the conclusions reached by Higgs and Joanny. They found that a PA carrying alternating positive and negative charges exhibits an usual coil-globule transition as the temperature decreases. This transition has all the properties of a regular $\theta$ point.

Wittmer et al. [14] pointed that the Debye-Hückel (DH) theory cannot be applied to an alternating PA, which they showed behaves as a "microdielectric" instead of microelectrolyte. As a result, they found that an alternating PA is similar to a neutral polymer, and the only role of electrostatic interactions is to renormalize the excluded volume contribution to the free energy. 
There are two possible classes of a neutral PA's: statistically neutral [15-17] or physically neutral [12]. In the first case, where the neutrality is only obtained when the average is done over the charge distribution, upon increasing the temperature, a swelling of the chain is observed in Monte Carlo (MC) simulations. This can be explained by the presence of a net charge of $\mathrm{O}(q \sqrt{N})$, where $q$ is the monomer charge and $N$ is the number of monomers on the chain. This produces charge fluctuations in different parts of the chain. The equally charged domains repel each other, forcing the chain to swell. The PA is stretched by the non-extensive energy cost of typical charge fluctuations [16].

In the case of physically neutral non-alternating PA, the situation remains unclear. In this paper, we shall analyze this case in the framework of the DH theory, combined with the Bjerrum's idea of ion association [18,19], and with Flory's affine network theory [5]. We shall explore the question of existence and nature of possible conformational phase transitions in a neutral polyampholyte.

In order to address this question we will show that strong electrostatic interaction between monomers leads to a formation of dipoles (or intermolecular bridges) [20]. This has two major effects: first, since the total number of monomers is fixed, each pair formation implies a decrease in the $\mathrm{DH}$ attractive energy; second, it transforms the linear chain into a network of functionality 4 . We will show that this implies that in the low temperature region, where the dipoles are most favorable, PA resembles an affine network, or a microgel [20]. As the temperature is raised, there is a collapse transition from this state to a dilute globular state usually called microelectrolyte [20]. This behavior is very different from the one found for usual neutral polymers, where short-range interactions play the dominant role. The nature of the microgel state will be investigated. Different scaling regimes for the low temperature state are obtained as a function of the quality of solvent.

The paper is organized in four sections. In Section 2 we first give a brief review of the Flory-Huggins model for an uncharged single chain. Then we summarize the DH theory augmented by the Bjerrum's idea of ion association for a simple electrolyte. Finally, in Section 3, we present our model that combines both ideas within the Flory's affine network theory. From this analysis, two conditions for the thermal equilibrium of the chain emerge. On the basis of these equations, we derive the full phase-diagram.

Various scenarios for different values of parameters of the model are then discussed. Conclusions are presented in Section 4.

\section{Introductions to the model}

Our model for a polyampholyte combines the concept of an affine network of rubber elasticity with a theory of simple electrolyte. In order to give a clear presentation we first review the two theories: the Flory-Huggins-De Gennes [2-4] theory of coil-globule transition in a neutral polymer and the theory of three-dimensional $(3 d)$ Coulomb gas $[18-21]$. 


\subsection{An uncharged chain}

A simple model for a single polymer, introduced by Flory and Huggins and later modified by De Gennes [2-4,22], assumes that a chain is made of $N$ monomers of diameter $a$. If $R$ measures the mean end-to-end distance occupied by the polymer, one can define the volume of a polymer as $V=4 \pi R^{3} / 3$, and the reduced density of monomers, $\rho^{*}=\rho a^{3}$, where $\rho=3 N /\left(4 \pi R^{3}\right)$. Here, $R$ is usually called radius of gyration of the polymer.

The free energy of this system would contain, in principle, two contributions: an attractive term due to the presence of the chain connecting the monomers and a repulsive term due to the excluded volume repulsion. The attractive force resembles the elastic interaction present in a strand of rubber and is, consequently, given by [2-5]

$$
\beta F_{\mathrm{el}} \approx \frac{R^{2}}{N} .
$$

For long chains, this radius is assumed to scale as

$$
R \approx a N^{v} .
$$

If the chain is made of non-interacting particles, the elastic contribution to the free energy given by Eq. (1) has a nontrivial thermodynamic limit, $(N \rightarrow \infty)$ only if $R$ scales with $v=\frac{1}{2}$. The same behavior is also obtained for the end-to-end distance of a random walk $[3,4,22]$.

In a real chain, however, the monomers interact between each other, and with the solvent. The interaction between monomers is due to the fact that no two monomers can occupy the same position in space. This leads to a repulsive contribution to the free energy. The monomer-solvent interaction can either be attractive or repulsive depending on the quality of solvent. If the solvent is good, its particles are attracted by the monomers, which leads to an effective repulsion between two different parts of the chain. In this case, the free energy due to the monomer-monomer and the monomer-solvent interactions can be written as a virial expansion given by

$$
\beta F_{h c} \approx \frac{1}{2} W_{1} N \rho+\frac{1}{2} W_{2} N \rho^{2} .
$$

The first term in Eq. (3) accounts for the two-body repulsive $\left(W_{1}>0\right.$, in a good solvent) interaction, while the second one represents the three-body contribution $\left(W_{2}>0\right.$, always).

Now, the free energy, obtained from adding the elastic and the hard core contributions, has a well defined minimum if the radius of gyration scales like Eq. (2) with $v=\frac{3}{5}$, indicating that the selfavoiding polymer is more stretched than a coil, i.e., is in an extended state [22-24].

If the solvent is bad, its particles repel the monomers and thus forcing them closer together, leading to an effective attraction. This effect can in principle be represented by an attractive interaction, $\Xi(T)$, that renormalizes the coefficient of the two body term, $\tilde{W}_{1}=W_{1}-\Xi(T)$. 
At high temperature, the polymer configurations are still dominated by the strong excluded volume interaction, $W_{1}$ and, consequently, the second virial coefficient is positive. For long chains the elastic term is balanced by this repulsive interaction and the size of the polymer scales as in a good solvent

$$
R \approx a N^{3 / 5} .
$$

As the temperature is decreased, the value of $\Xi$ increases and, consequently, the second virial coefficient, $W_{1}$, becomes zero or even negative. When $W_{1}=0$, the third virial term has to be included. In this case, the three-body term balances the attractive elastic contribution and the polymer behaves like a coil with

$$
R \approx a N^{1 / 2} .
$$

At lower temperatures, the interactions between two monomers become attractive. Competition between the two-body and the three-body terms leads to a collapsed structure with

$$
R \approx a N^{1 / 3} .
$$

Of course, this picture is valid only if the interactions between the monomers are of elastic or short ranged nature. In the next section we will introduce another type of interaction that will modify significantly the behavior described above.

\subsection{An electrolyte}

Before proceeding any further with the polymer analysis, we shall review the results for the phase separation in a restrictive primitive model (RPM) of an electrolyte [21]. The model consists of $N=\rho V$ hard spheres of diameter $a$, where half of the particles carry positive charges $+q$ and the other half carry negative charges $-q$. The whole system is confined in a volume $V$. The solvent is treated as a uniform medium of a dielectric constant $D$. The interaction potential between two ions $(i, j)$ is

$$
\phi_{i j}= \begin{cases}\frac{q_{i} q_{j}}{D r_{i j}}, & r_{i j} \geqslant a, \\ \infty, & r_{i j}<a .\end{cases}
$$

The Helmoltz free energy of this system exhibits an attractive electrostatic contribution due to the charge density fluctuations. For the RPM, this energy, in the leading order, can be approximated by the Debye-Hückel limiting law given by

$$
\beta F_{\mathrm{LL}}=-V \frac{\kappa^{3}}{12 \pi},
$$

where $\beta \equiv 1 / k_{\mathrm{B}} T, k_{\mathrm{B}}$ is the Boltzman constant and

$$
\kappa^{2}=4 \pi \frac{q^{2} \rho}{D k_{\mathrm{B}} T}
$$


is the inverse of the screening length, $\xi_{\mathrm{D}}=1 / \kappa$. The expression, Eq. (8) is satisfactory only when $\kappa \rightarrow 0$, in other words, when the density is low and the temperature is high. For studying Coulombic phase separation which occurs at $\kappa a \approx 1$, this expression is insufficient. It predicts a critical temperature that is higher than the $\mathrm{MC}$ results by a factor from 9 to $10[25,26]$. In order to improve this result, one has to go back to the full $\mathrm{DH}$ form for the electrostatic free energy given by

$$
\beta F_{\mathrm{DH}}=\frac{N}{k_{\mathrm{B}} T^{*}(\kappa a)^{2}}\left[\kappa a-\frac{(\kappa a)^{2}}{2}-\ln (\kappa a+1)\right] .
$$

In the above expression $T^{*}=k_{\mathrm{B}} T D a / q^{2}$. This form, apparently, remains valid even when the limiting law brakes down.

The use of the full DH free energy produces a critical temperature in close agreement with $\mathrm{MC}$, but the critical density is too small by a factor of 6 . To understand this, we have to go back and reexamine the original $\mathrm{DH}$ derivation.

Debye and Hückel focused their attention on one ion. The electrostatic potential in the region of this ion satisfies a Poisson-Boltzman equation (PB) $[18,27]$ given by

$$
\nabla^{2} \phi=-\frac{4 \pi}{D} \rho_{q}
$$

Debye and Hückel assumed that other ions will distribute themselves around the central ion according to the Boltzman distribution $\rho_{q}=q \rho_{+} \exp (-\beta q \phi)-q \rho_{-} \exp (\beta q \phi)$, where $\rho_{+}=\rho_{-}=\frac{1}{2} \rho$. In order to solve this equation, Debye and Hückel proceed to linearize the exponential. This approximation, besides simplifying the calculations, restores the electrostatic self consistency which the full non-linear PB equation lacks [27]. The process of linearization, however, lowers the effective weight of configurations in which oppositely charged ions come into a close proximity of each other. Bjerrum [19] suggested that these dipolar pair configurations can be reintroduced into the theory by allowing a chemical equilibrium to exist between the free charges and the dipoles. This is established by requiring that

$$
\mu_{+}+\mu_{-}=\mu_{2}
$$

where

$$
\mu_{ \pm} \equiv \frac{\partial F}{\partial N_{ \pm}}, \quad \mu_{2} \equiv \frac{\partial F}{\partial N_{2}}
$$

are the chemical potentials of the negative, positive charges, and of the dipolar pairs. The combination of the DH theory and the Bjerrum association does not change the critical temperature, but gives a critical density that is only 1.5 times larger than $\mathrm{MC}$.

The introduction of dipole-ion interaction brings the coexistence curve in an excellent agreement with MC $[28,29]$. Furthermore, the theory can be easily extended to general dimensions and in particular [29,30], reproduces the Kosterlitz-Thouless [31] result of an infinite order line of metal-insulator transitions in two dimensions, with an additional prediction that this line terminates in a tricritical point. 
Given that the above theory was quite successful to explain the behavior of a gas of charged particles, it is then natural to ask if the mechanism of dipolar formation is also relevant for the polyampholytes. The preliminary reports of our findings has been given in Ref. [20]. Below we shall present the details of our calculations.

\section{The polyampholyte}

\subsection{The model}

We consider a neutral polyampholyte chain of $N$ spherical monomers, each of diameter $a$. The monomers are of three types, $N_{0}$ neutral monomers, $\frac{1}{2}\left(N-N_{0}\right)$ positive monomers, and $\frac{1}{2}\left(N-N_{0}\right)$ negative monomers. The charged monomers can associate forming intermolecular bridges (dipoles). After thermal equilibrium is established, the chain resembles an affine network with the crosslinks of functionality four. Three distinct structures are found along the chain: neutral monomers, charged monomers and dipoles. Now, defining $f$ as the number fraction of non-neutral monomers and assuming that a fraction $x$ of such charges form dipoles, we find

$$
\begin{aligned}
& N_{0} \equiv(1-f) N, \quad \text { neutral , } \\
& N_{1} \equiv f N(1-2 x) \text {, monopoles , } \\
& N_{2} \equiv f N x, \quad \text { dipoles. }
\end{aligned}
$$

Within our model, the whole chain can be viewed as a network, made of segments connected at the dipolar pairs by the crosslinks of functionality 4 (see Fig. 1). Note that, even if the individual segments of the chain might exhibit a nonzero charge, the whole network is neutral.

Following the same procedure introduced in Section 2.1 , we consider the polymer confined inside a sphere of volume, $V=\frac{4}{3} \pi R^{3}$. The density of monomers is then given by $\rho=\rho^{*} a^{3}$ where $\rho^{*}=3 \alpha^{-3} /(4 \pi \sqrt{N})$. $\alpha \equiv R / R_{0}$ measures the relative extension of the chain with respect to an ideal chain with radius of gyration $R_{0} \approx a \sqrt{N}$. The densities of neutral monomers, the monopoles and the dipoles are respectively

$$
\rho_{0} \equiv(1-f) \rho, \quad \rho_{1} \equiv f(1-2 x) \rho, \quad \rho_{2} \equiv f x \rho .
$$

The total free energy of the chain is constructed as a sum of the most relevant contributions:

(a) an elastic energy due to the elongation and contraction of the active strands,

(b) an entropic energy that is due to the mixing of different types of particles,

(c) a repulsive energy due to the excluded volume effects,

(d) and the attractive energy due to the net electrostatic interactions.

According to the affine network theory of rubber elasticity $[2,5,32,33]$, the elastic free energy is

$$
\beta F_{\mathrm{el}}=\frac{v}{2}\left(\lambda_{x}^{2}+\lambda_{y}^{2}+\lambda_{z}^{2}-3\right)-\mu_{\mathrm{J}} \ln \alpha,
$$




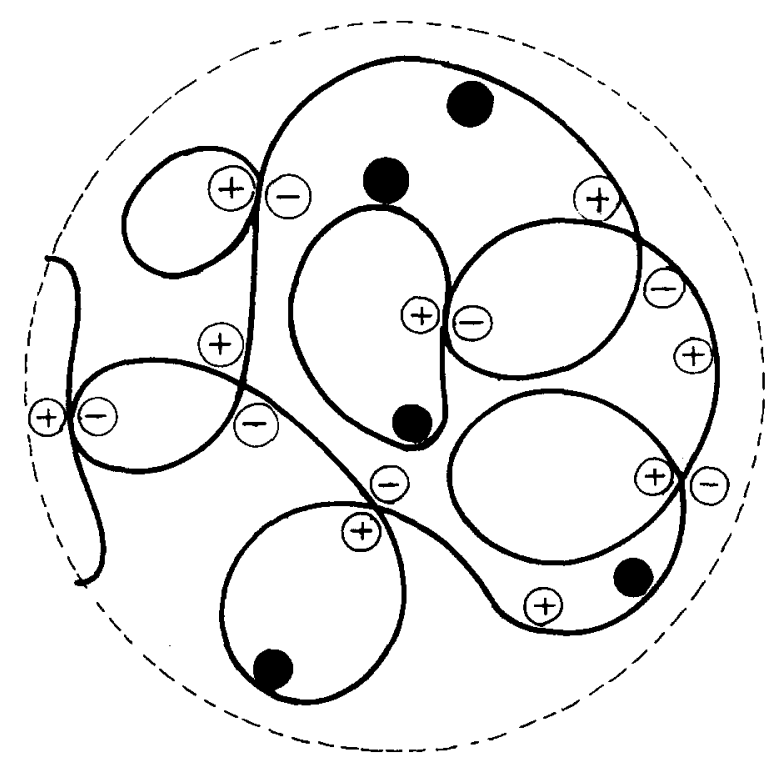

Fig. 1. Globular state of a polymer in a solvent is regarded as a network of functionality 4 . The chain, made of positive $(+)$, negative ( - ) and neutral (black) monomers of diameter $a$, is confined to a region of volume $V=3 /\left(4 \pi R^{3}\right)$ where the radius of gyration is given by $R \propto a N^{v}$.

where $v$ is the number of elasticly active strands in the network, $\mu_{\mathrm{J}}$ is the number of junctions, and $\lambda_{x}, \lambda_{y}$ and $\lambda_{z}$ are the principal extension ratios measured relative to the dimensions of the chain in an unstrained state. In the present case, we assume a uniform expansion $\lambda_{x}=\lambda_{y}=\lambda_{z}=\alpha$. From geometrical considerations we find that the number of junctions is $\mu_{\mathrm{J}}=N_{2}+1$, where the extra one comes from fixing one monomer at the origin. Similarly, the number of elasticly active strands is $v=2 N_{2}+1$. The elastic free energy is then [33]

$$
\beta F_{\text {el }}=\frac{3}{2}\left(\alpha^{2}-1-2 \ln \alpha\right)+3 N_{2}\left(\alpha^{2}-1-\ln \alpha\right) .
$$

One might note that, in the absence of dipoles, this is equivalent to the elastic energy in an uncharged polymer, when the full theory of elasticity is taken into account [7]. When $\alpha$ is large, it can be approximated by Eq. (1).

The polymer resembles a mixture of an electrolyte and a neutral gas occupying a volume $V$. However, there is an important difference. While in the RPM ions are free to move independently, the monomers of a PA are constrained by the bonds that maintain an overall integrity of the chain. Nevertheless, we must still account for the increase in the free energy due to the mixing of particles of different types [33,34].

If the bonds were cut, the ideal free energy of the resulting "gas" of positive, negative, neutral monomers, and dipolar pairs would be given by

$$
\beta F_{\text {ideal }}=\sum_{j}\left[N_{j} \ln \frac{\rho_{j} A^{3 k_{j}}}{\zeta_{j}}-N_{j}\right],
$$


where $\zeta_{i}$ is the molecular partition function of the species $i$, while $\Lambda_{i}=h / \sqrt{2 \pi \bar{m}_{i} k_{\mathrm{B}} T}$ is the mean thermal wavelength, $\bar{m}_{i}$ is the geometric mean mass and the parameter $k_{i}$ is the number of "atoms" in each "molecule" of the resulting gas. In our case, $k_{0}=k_{ \pm}=1, k_{2}=2$ and the geometric masses are $\bar{m}_{0}=\bar{m}_{ \pm}=\bar{m}_{2}=m$. However, since all of the monomers are connected by bonds and, therefore, are not free to move independently, in order to obtain the correct increase in the free energy, it is necessary to subtract from this expression the free energy of an ideal gas made of just one type of particles. This leads to an energy of the mixture $[33,34]$

$$
\beta F_{\text {mix }}=\sum_{j}\left[N_{j} \ln \frac{\rho_{j} \Lambda^{3 k_{j}}}{\zeta_{j}}-N_{j}\right]-\left[N \ln \frac{\rho \Lambda^{3}}{\zeta}-N\right],
$$

that can be expressed as

$$
\beta F_{\text {mix }}=N_{0} \ln \frac{\rho_{0}}{\zeta_{0}}+N_{1} \ln \frac{\rho_{1}}{2 \zeta_{1}}+N_{2} \ln \frac{\rho_{2}}{\zeta_{2}}-N \ln \frac{\rho}{\zeta}+N_{2} .
$$

Since all the monomers are hard spheres with no internal structure the molecular partition functions for the monopoles and neutral particles are given by $\zeta=\zeta_{ \pm}=\zeta_{0}=1$. For the dipoles the molecular partition function is

$$
\zeta_{2}=\int_{a}^{c} \mathrm{e}^{a / T^{*} r} r^{2} \mathrm{~d} r \equiv K(T)
$$

For the upper cutoff $c$, we use the Bjerrum's choice, $c=a / 2 T^{*}$, which corresponds to the inflection point of the integral as a function of $c[18,19,21,28]$. Then, the value of the association constant is found to be [35]

$$
K\left(T^{*}\right)=\frac{2 \pi a^{3}}{3\left(T^{*}\right)^{3}}\left[\operatorname{Ei}\left(\frac{1}{T^{*}}\right)-\operatorname{Ei}(2)+\mathrm{e}^{2}\right]-\frac{2 \pi a^{3}}{3} \mathrm{e}^{1 / T^{*}}\left[2+\frac{1}{T^{*}}+\frac{1}{\left(T^{\star}\right)^{2}}\right]
$$

This expression is in close agreement with the one obtained by Ebeling on the basis of a cluster expansion [36].

The electrostatic contribution to the free energy is given by Eq. (10) with $N$ and $\rho$ replaced by $N_{1}$ and $\rho_{1}$, the total number and density of free charges. While the excluded volume and the polymer-solvent interaction are given by Eq. (3).

\subsection{Thermodynamics of the system}

Using this model, we can now ask what are the equilibrium configurations of a polyampholyte. In order to answer this question we have to examine the Gibbs free energy of a PA in a solvent [37]. The Gibbs free energy is given by

$$
\mathscr{G}=F_{\mathrm{p}}+F_{\mathrm{s}}+P_{\text {out }} V-\mu_{\text {out }} N_{\mathrm{s}}
$$

where $F_{\mathrm{p}}=F_{\mathrm{el}}+F_{\mathrm{hc}}+F_{\text {mix }}+F_{\mathrm{DH}}$ is the Helmoltz free energy of the chain, $F_{\mathrm{s}}$ is the Helmoltz free energy of the solvent particles inside $V$ and $P_{\text {out }}$ and $\mu_{\text {out }}$ are, 
respectively, the pressure and the chemical potential of the solvent outside the volume $V$. In the simple case that we are studying, the polymer-solvent interaction is already taken into account in the second and the third virial coefficients of the excluded volume contribution of Eq. (3), therefore, in the above expression we treat the solvent as an ideal gas.

For a fixed number of charged monomers ( $f$ fixed), the Gibbs free energy is a function of three independent variables: the number of solvent particles inside the volume $V, N_{\mathrm{s}}$, the volume $V$ itself, and the fraction of free charges along the chain, $x$. The equilibrium configuration is, then, obtained by a minimization of Eq. (23) with respect to these parameters. The minimization with respect to the volume leads to

$$
\frac{\partial \beta F_{\mathrm{p}}}{\partial V}-P_{\mathrm{s}}+P_{\mathrm{out}}=0
$$

where

$$
\frac{\partial \beta F_{\mathrm{s}}}{\partial V} \equiv-P_{\mathrm{s}},
$$

has been used. Since we are assuming no interaction between solvent and polymer, $P_{\mathrm{s}}=P_{\text {out }}$ and, consequently, $\partial \beta F_{\mathrm{p}} / \partial V=0$. Equivalently,

$$
\begin{aligned}
\frac{\partial \beta F}{\partial \alpha}= & 3\left(\alpha-\frac{1}{\alpha}+2 N x f \alpha\right)-\frac{3}{2} N \alpha^{-1} W_{1} \rho-3 N \alpha^{-1} W_{2} \rho^{2} \\
& -\frac{3 N f(1-2 x) \alpha^{-1}}{2 T^{*} \kappa a}\left[2 \frac{\ln (\kappa a+1)}{\kappa a}-\frac{\kappa a+2}{\kappa a+1}\right]=0 .
\end{aligned}
$$

Minimization with respect to the solvent particles leads to the condition $\mu_{\mathrm{s}}=\mu_{\mathrm{out}}$, where

$$
\mu_{\mathrm{s}}=\frac{\partial \beta F_{\mathrm{s}}}{\partial N_{\mathrm{s}}} .
$$

Since the number of monopoles and dipoles are functions of $x$, minimization of the free energy with respect to this fraction can be written as

$$
\frac{\partial \beta F_{\mathrm{p}}}{\partial x} \equiv \frac{\partial F}{\partial N_{+}} \frac{\partial N_{+}}{\partial x}+\frac{\partial F}{\partial N_{-}} \frac{\partial N_{-}}{\partial x}+\frac{\partial F}{\partial N_{2}} \frac{\partial N_{2}}{\partial x}=0 .
$$

Using now the definitions Eq. (13) and Eq. (14), we find

$$
\frac{\partial \beta F_{\mathrm{p}}}{\partial x} \equiv N f\left(\mu_{-}+\mu_{+}-\mu_{2}\right)=0 .
$$

This is the chemical equilibrium equation, Eq. (12). Using the explicit form for the free energy, the chemical potentials are,

$$
\mu_{1}=\mu_{ \pm} \equiv \frac{\partial \beta F}{\partial N_{ \pm}}=W_{1} \rho+\frac{3}{2} W_{2} \rho^{2}-\frac{\kappa a}{2 T^{*}(\kappa a+1)}+\ln \frac{\rho_{1}}{\zeta_{1}}-\ln \frac{\rho}{\zeta},
$$




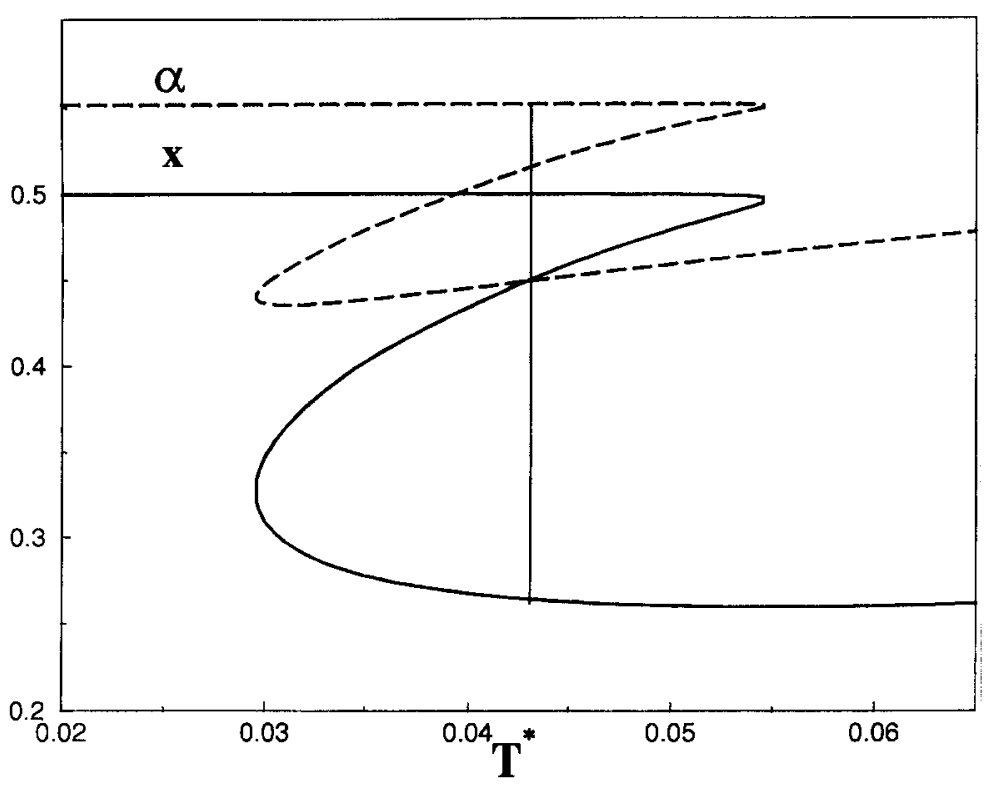

Fig. 2. Behavior of $x$ and $\alpha$ as a function of temperature, $T$ for $N=100, f=1$ and $W_{1}=4 \pi a^{3} / 3$ corresponding to the second virial coefficient for the gas of hard spheres. The non-single-valued dependence of $\alpha$ on the temperature indicates the presence of first order phase transition. The transition, indicated by a vertical line, occurs at the temperature $\bar{T}^{*}$ for which the two branches of free energy have the same value.

$$
\mu_{2} \equiv \frac{\partial \beta F}{\partial N_{2}}=3\left(\alpha^{2}-1-\ln \alpha\right)+2 W_{1} \rho+3 W_{2} \rho^{2}+\ln \frac{\rho_{2}}{\zeta_{2}}-2 \ln \frac{\rho}{\zeta} .
$$

The law of mass action, $\mu_{-}+\mu_{+}=\mu_{2}$, can now be written as

$$
\frac{x}{(1-2 x)^{2}}=\frac{3 f K(T)}{16 \pi a^{3} \sqrt{N}} \exp \left[-\frac{\kappa a}{T^{*}(\kappa a+1)}+3-3 \alpha^{2}\right] \text {. }
$$

The equilibrium configuration of the polyampholyte is given by the solutions of Eqs. (26) and (32). For $N=100, f=1$ and $W_{1}=\frac{4}{3} \pi a^{3}$ the values of $x$ and $\alpha$, as a function of temperature, are shown in Fig. 2.

At high temperatures, the relative size of the chain, $\alpha$, is small and decreases with the temperature. The value of $\alpha$ decreases as $N$ is increased. The fraction $x$, in this state, is quite small, indicating that few dipolar pairs have formed.

In order to find what is the state of the chain at high temperature, we have to explore how the radius of gyration scales with $N$ as $N \rightarrow \infty$. At high temperatures (small $\kappa a$ ), Eq. (26) can be approximated by

$$
-3 W_{1} \rho+\frac{f}{T^{*}} \kappa a=0,
$$

which leads to a radius of gyration that scales as

$$
R \approx a_{0} N^{1 / 3},
$$


where $a_{0}=3 a T^{*}\left(W_{1}^{*}\right)^{2 / 3} /\left((4 \pi)^{2 / 3} f\right)$ and $W_{1}^{*} \equiv W_{1} a^{-3}$. This collapsed state is less dense than the collapsed state of an usual neutral polymer for which $a_{0}=\left(3 W_{2} /\right.$ $\left.\left(2 \pi W_{1}\right)\right)^{1 / 3}[3,12]$.

As the temperature is lowered the scenario changes drastically. Since the ion association is energetically favorable, positive and negative charges come close together, forming intermolecular bridges. Thus, for low temperatures, almost all charged monomers are paired up and $x \approx \frac{1}{2}$. Consequently, since the number of free (unpaired) monopoles is small, the electrostatic energy given by Eq. (10) becomes negligible. The attractive elastic energy, given by Eq. (17), balances the hardcore repulsion. The chain, however, unlike a neutral homopolymer, is made of strands connected by crosslinks of functionality four (see Fig. 1).

The equilibrium configuration of this network is given by the minimization equation in the absence of monopoles, $x \rightarrow \frac{1}{2}$. Eq. (26) then simplifies to

$$
3\left(\alpha^{2}-1+N f \alpha^{2}\right)-\frac{3}{2} N W_{1} \rho-3 N W_{2} \rho^{2}=0 .
$$

If the solvent is good $\left(W_{1}>0\right)$, the dominant contributions (for large $N$ ) in Eq. (35) are

$$
3 N f \alpha^{2}-\frac{3}{2} N W_{1} \rho=0,
$$

this leads to a radius of gyration given by

$$
R \approx a_{1} N^{2 / 5}
$$

with $a_{1} \approx\left(3 W_{1}^{*} /(8 \pi f)\right)^{1 / 5} a$. Consequently, the microgel is an extended state. Comparing the exponent $v=\frac{2}{5}$ with the usual Flory exponent $v=\frac{3}{5}$, given by Eq. (4), we note that, due to the presence of crosslinks, the network is smaller than the usual neutral polymer.

If the quality of the solvent is decreased, the second virial coefficient $W_{1}$ becomes zero, and the leading order terms in Eq. (35) are

$$
N f \alpha^{2}-N W_{2} \rho^{2}=0 .
$$

The radius of gyration is then,

$$
R \approx a_{2} N^{3 / 8}
$$

where $a_{2} \approx\left(9 W_{2}^{*} /\left(16 \pi^{2} f\right)\right)^{1 / 8} a$, where $W_{2}^{*} \equiv W_{2} a^{-6}$. The microgel assumes a conformation that resembles a coil, smaller than the usual Flory coil for which $v=\frac{1}{2}$.

If the quality of solvent is decreased further, the second virial coefficient $W_{1}$ becomes negative. In the limit of very long chains, the main contributions in Eq. (35) are the second and the third virial coefficients, namely

$$
-\frac{3}{2} N W_{1} \rho-3 N W_{2} \rho^{2}=0
$$




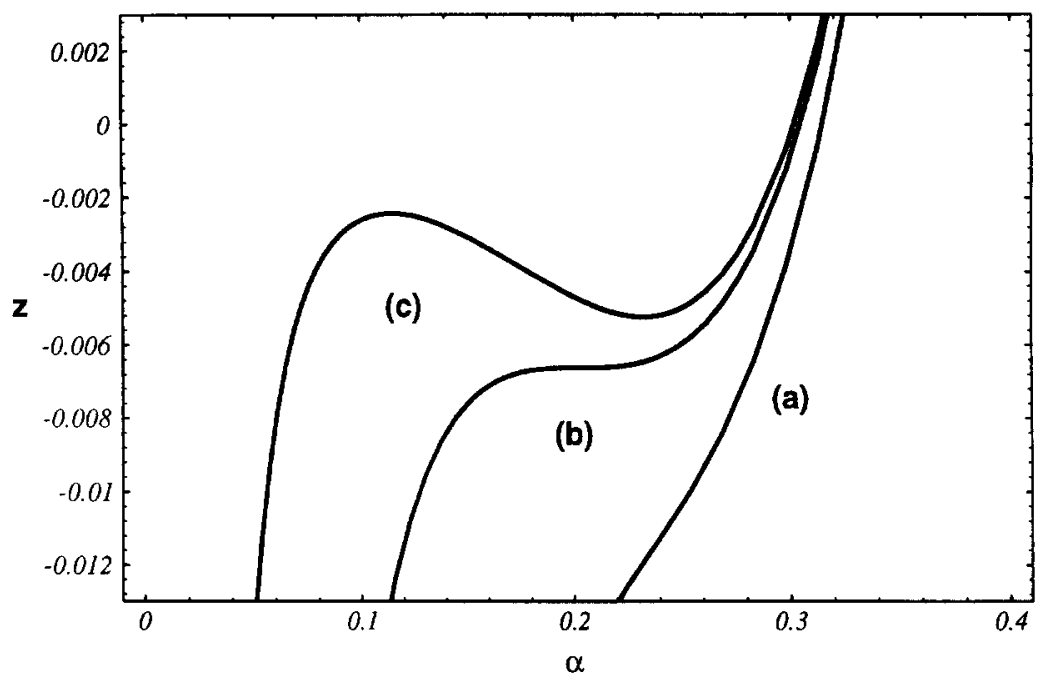

Fig. 3. $z$ as a function of $\alpha$ for: (a) $W_{2}>\bar{W}_{2}$, (b) $W_{2}=\bar{W}_{2}$ and (c) $W_{2}<\bar{W}_{2}$.

The network is, therefore, in a dense globular state for which

$$
R \approx a_{3} N^{1 / 3}
$$

where $a_{3}=\left\{3 W_{2} /\left(2 \pi\left|W_{1}\right|\right)\right\}^{1 / 3}$.

The "crossover" between these scaling regimes can be obtained from Eq. (35), namely

$$
\alpha^{5}-\alpha^{3}+N f \alpha^{5}-\frac{9 W_{2}^{*}}{(4 \pi)^{2} \alpha^{3}}=z,
$$

where $z \equiv 3 \sqrt{N} W_{1}^{*} /(8 \pi)$ (see Fig. 3). For a fixed value of $W_{2}$ and positive values of the second virial coefficient, this equation has one possible value of $\alpha$ for each value of $z$. The radius of gyration of this solution scales as Eq. (37), indicating that the polymer is extended.

For $W_{2}<81 \pi^{2} /\left(2000(N f+1)^{3}\right) a^{6}$ and $z<0$, Eq. (42) has three possible solutions for $\alpha$. One corresponds to extended states and the other corresponds to a dense globular state that scales as Eq. (41). Connecting these two scaling regimes, there is an intermediate state. In order to define which solution leads to the minimum free energy configuration, the free energies of these three states must be calculated and compared. For $x=\frac{1}{2}$, the free energy associated with an isolated chain is given by

$$
\begin{aligned}
\beta \mathscr{G}= & \frac{3}{2}\left(\alpha^{2}-1-2 \ln \alpha\right)+3 N_{2}\left(\alpha^{2}-1-\ln \alpha\right)+\frac{N f}{2} \ln \frac{\rho f}{2 K(T)} \\
& +N(1-f) \ln \rho(1-f)-N \ln \rho+\frac{N f}{2}+\frac{N W_{1} \rho}{2}+\frac{N W_{2} \rho^{2}}{2} .
\end{aligned}
$$

This corresponds to the first term $F_{\mathrm{p}}$ in Eq. (23) since the other contributions just add to zero. 
For $W_{1}>-\bar{W}_{1}$, the extended state (microgel) has the lowest free energy, while the dense globular state has the lowest energy for $W_{1}<-\bar{W}_{1}$. At $W_{1}=-\bar{W}_{1}$, there is a first-order phase transition between them (see Fig. 3). This transition, present only at low temperatures, does not explicitly depend on the value of $T^{*}$ but is only a function of the quality of solvent. The intermediate state is always metastable. In the limit of $N \rightarrow \infty$ the phase transition between the microgel and the dense globular state occurs at $W_{1}=\bar{W}_{1}=0$ and the radius of gyration of the polymer scales as Eq. (37). This behavior resembles a tricritical point [3].

Now, connecting the high and low temperature behaviors (see Fig. 2), there is a range of temperatures for which both $x$ and $\alpha$ are not single valued functions and three possible solutions can be found.

Comparing the free energies of the three solutions, we find that the solution with lower value of $x$ is the minimum for temperatures $T>\bar{T}$, while the solution with $x \approx \frac{1}{2}$ is the minimum for $T<\bar{T}$ (see Fig. 2). At a temperature $T=\bar{T}$, there is a first-order phase transition from the high temperature dilute globular state, the microelectrolyte, to one of the possible states present at low temperatures. For a finite chain, the network assumes one of the following conformations: an extended state (microgel), a dilute globular state (microelectrolyte) or a fully collapsed state (see Fig. 4).

This result was checked for different values of the total number of monomers, $N$, and for different values of the fraction of charged monomers, $f$.

No qualitative change is observed when these two parameters are varied. The increase in $N$ leads to a decrease in the transition temperature, since, in that case, the resulting increase in entropy favors the distraction of the ordered microgel phase. Fig. 5 plots the transition temperature, $\bar{T}^{*}$ versus the total number of monomers, $N$ [20].

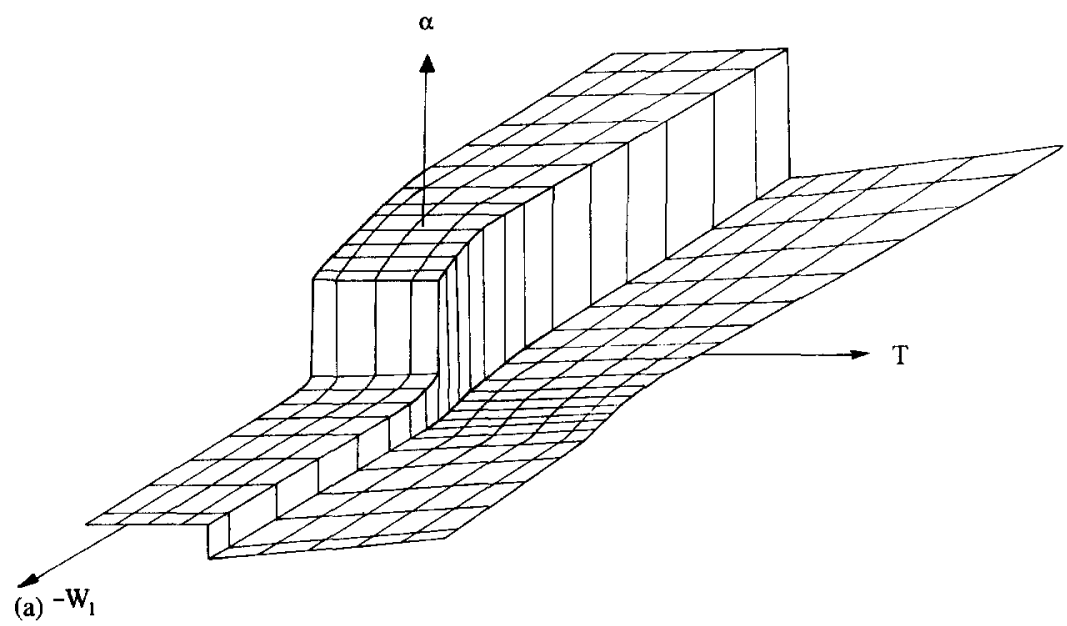

Fig. 4. Schematic phase-diagram indicating the behavior of $\alpha$ as a function of temperature and of the second virial coefficient, $W_{1}$, as it varies from positive to negative values for: (a) $W_{2}<\bar{W}_{2}$ and (b) $W_{2} \geqslant \bar{W}_{2}$. 


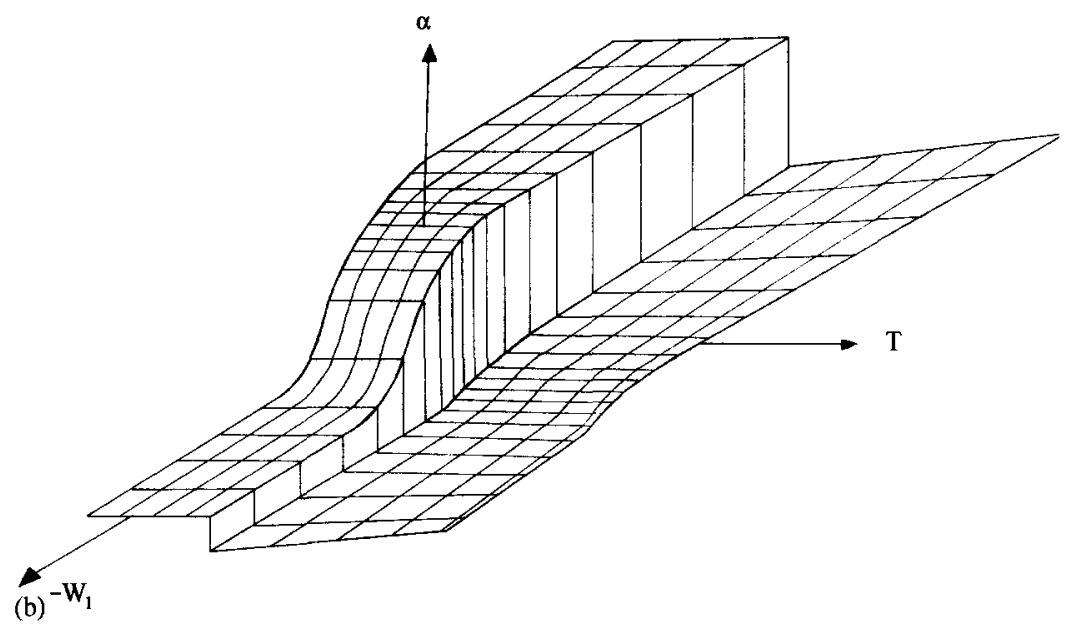

Fig. 4. Continued.

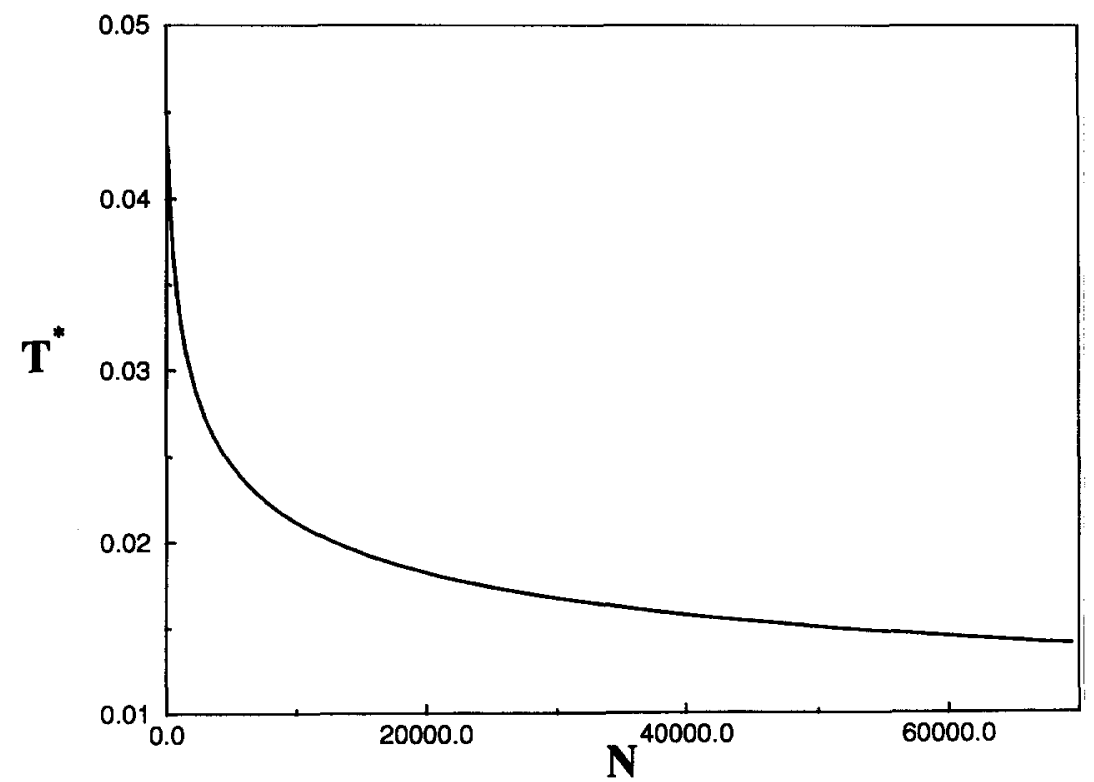

Fig. 5. Dependence of the transition temperature on the length of the polyampholyte for $f=1$ and $W_{1}=\frac{4}{3} \pi a^{3}$. For large $N$, the system is close to algebraic decaying.

As the fraction of charged monomers, $f$, is decreased, the transition temperature increases.

The first-order transition is also not drastically affected by the quality of solvent. We found that the transition temperature $\bar{T}^{*}$ varies continuously as $W_{1}$ changes from positive to negative values as is shown in Fig. 4. 


\section{Conclusions}

In this paper we have analyzed the behavior of a PA in the framework of the DebyeHückel Bjerrum-Flory (DHBjF) theory. We find that at reduced temperature $T^{*}=\bar{T}^{*}$, there is a first-order phase transition from a high temperature dilute globular state to a low temperature microgel state.

The microgel, present at low temperatures, can be either extended or fully collapsed depending on the quality of the solvent. The transition between the extended and collapsed state as $N \rightarrow \infty$ resembles a tricritical point.

In order to verify that the first-order transition between the microgel and the microelectrolyte is actually present in real systems, we have to check if the transition temperature is consistent with the properties of real solvents. The temperature, $\bar{T}$, is strongly dependent on the value of the dielectric constant of the solvent, $D$.

The aqueous solution at a room temperature has a dielectric constant $D=80 \varepsilon_{0}$ ( $\varepsilon_{0}$ is the permittivity of the vacuum). The transition temperature for a polymer with $N=100$, comes out to be $\bar{T} \approx 100 \mathrm{~K}$, if $a \approx 1 \AA$. Obviously, this transition cannot occur under normal laboratory conditions since the water is going to freeze before the transition happens! However, there are many organic solvents that have dielectric constants close to $20 \varepsilon_{0}$ at $\bar{T} \approx 300 \mathrm{~K}$. For these solvents, the transition from the high temperature microelectrolyte state to the low temperature microgel should occur at around the room temperature.

Although we have demonstrated that the absolute minimum of the free energy at low temperatures corresponds to a microgel, the real PA molecule is a complex system with possibly very many metastable states separated by energy barriers. Depending on the heights of these barriers, as the temperature is lowered, PA can get stuck in one of the metastable minima. The possibility of a glass transition must then be kept in mind.

In the future work we shall explore the effects of the presence of $1: 1$ salt on the conformational properties of a PA [40].

\section{Acknowledgements}

This work was supported in part by CNPq-Conselho Nacional de Desenvolvimento Científico e Tecnológico and FINEP-Financiadora de Estudos e Projetos, Brazil.

\section{References}

[1] N. Go and H. Taketomi, Proc. Natl, Acad. Sci. USA, 75 (1978) 559; T. Garel and H. Orland, Europhys. Lett. 6 (1988) 307.

[2] P.J. Flory, Principles of Polymer Chemistry (Cornell University Press, Ithaca, NY, 1953).

[3] P.G. de Gennes, J. Phys. Lett. 36 (1975) L-55.

[4] P.G. de Gennes, Scaling Concepts in Polymer Physics, (Cornell University Press, Ithaca, NY, 1979).

[5] P.J. Flory, Proc. R. Soc. London 351 (1976) 351. 
[6] P. Pfeuty, R.M. Velasco and P.G. De Gennes, J. Phys. Lett. 38 (1977) L-5.

[7] P.G. de Gennes, P. Pincus, R.M. Velasco and F. Brochard, J. Phys. (Paris) 1 (1976) 1461.

[8] J.F. Joanny and P. Pincus, Polymer 21 (1980) 274.

[9] G.S. Manning, J. Chem. Phys. 5 (1969) 924, 3249.

[10] Y. Levin, Europhys. Lett. 34 (1996) 405.

[11] S.F. Edwards, P.R. King and P. Pincus, Ferroelectrics 30 (1980) 7.

[12] P.G. Higgs and J.F. Joanny, J. Chem. Phys. 94 (1991) 1543.

[13] J.M. Victor and J.B. Imbert, Europhys. Lett. 24 (1993) 189.

[14] J. Wittmer, A. Johner and J.F. Joanny, Europhys. Lett. 24 (1993) 263.

[15] Y. Kantor, M. Kardar, Europhys. Lett. 14 (1991) 421. Y. Kantor, H. Li and M. Kardar, Phys. Rev. Lett. 69 (1992) 61.

[16] Y. Kantor, M. Kardar and H. Li, Phys. Rev. E 49 (1994) 1381.

[17] Y. Kantor and M. Kardar, Europhys. Lett. 28 (1994) 169. Y. Kantor and M. Kardar, Phys. Rev. E 51 (1995) 1299.

[18] P.W. Debye and E. Hückel, Phys. Z. 24 (1923) 185; For a good exposition see D.A. MacQuarrie, Statistical Mechanics (Harper and Row, New York, 1976) Chap. 15.

[19] N. Bjerrum, Kgs. Dan. Vidensk. Selsk. Mat.-fys. Medd. 7 (1926) 1.

[20] Y. Levin and M.C. Barbosa, Europhys. Lett. 31 (1995) 513.

[21] For a review: Y. Levin and M.E. Fisher Physica A, 225 (1996) 164.

[22] P.G. des Gennes, Phys. Lett. A38 (1972) 339.

[23] J. des Cloizeaux, J. Phys. (Paris) 36 (1975) 281.

[24] J. des Cloizeaux, J. Phys. (Paris) 37 (1976) 431.

[25] G.R. Stell, K.C. Wu and B. Larsen, Phys. Rev. Lett. 37 (1976) 1369.

[26] J.M. Valleau, J. Chem. Phys. 95 (1991) 584. A.Z. Panangiotopoulos, Fluid Phase Equil. 76 (1992) 97; J.-M. Caillol, J. Chem. Phys. 100 (1994) 2161; G. Orkoulas and A.Z. Panangiotopoulos, J. Chem. Phys. 101 (1994) 1452.

[27] L. Onsager, Chem. Rev. 13 (1933) 73.

[28] M.E. Fisher and Y. Levin, Phys. Rev. Lett. 71 (1993) 3826; M.E. Fisher, J. Stat. Phys. 75 (1994) 1.

[29] Y. Levin, X.-J. Li and M.E. Fisher, Phys. Rev. Lett. 73 (1994) 2716; M.E. Fisher, X.-J. Li and Y. Levin, J. Stat. Phys. 79 (1995) 1.

[30] Y. Levin, X.-J. Li and M.E. Fisher, to be published.

[31] J.M. Kosterlitz and D.J. Thouless, J. Phys. C 6 (1973) 1181.

[32] G. Allen, Proc. R. Soc. Lond. A 351 (1976) 381.

[33] F. Tanaka and H. Ushiki, J. Chem. Phys. 84 (1986) 5925.

[34] I.M. Lifshitz, A.Yu. Grosberg and A.R. Khokhlov, Sov. Phys. JETP 44 (1976) 855.

[35] H. Yokoyama and H. Yamatera, Bull. Chem. Soc. Japan 48 (1975) 1770; Chem. Lett. 1973 (1975) 337.

[36] W. Ebeling, Z. Phys. Chem. 238 (1968) 400.

[37] All along there has been quite a lot of confusion about what energy is to be minimized. It is important to remember that $F$ is the Helmoltz free energy. In general the equilibrium configuration does not correspond to the minimum of the Helmoltz free energy. Only in the case when solvent particles are treated as an ideal gas can this be justified, see discussion after Eq. (23). For interacting solvents one must always work with a Gibbs free energy.

[38] R.R. Singh and K.S. Piltzer, J. Chem. Phys. 92 (1990) 6775.

[39] T.K. Narayanan and S. Pitzer, Phys. Rev. Lett. 73 (1994) 3002.

[40] A. Diehl, M.C. Barbosa and Y. Levin, in preparation. 